action of ubiquitinated $\mathrm{I} \kappa \mathrm{B}$ with the proteasome (after its dissociation from NF- $\mathrm{KB}$ ). These observations provide further evidence that the GA repeat works through proteasome inhibition, but several interesting questions remain. For example, the nature of the interaction between the GA repeat and proteasome requires definition, as does the exact mechanism of NF- $\mathrm{kB}$ inhibition, bearing in mind that the modified IкB $\alpha$ is able to dissociate from its complex with NF-kB after ubiquitination. The reason for the marked variability in the length of the GA repeat in different $\mathrm{EBV}$ strains is also intriguing.

Can the use of a GA repeat to inhibit I $\mathrm{KB} \alpha$ degradation ever be clinically useful? Previous studies have attempted to suppress NF- $\mathrm{kB}$ mediated inflammatory responses (for example in models of xenograft rejection $^{8}$ ) by overexpressing or mutating I $\mathrm{I} B \alpha$ to inhibit its degradation. Unfortunately, this can also sensitize cells to TNF $\alpha$-mediated apoptosis, a process that can be inhibited by NF- $\kappa B$ activation ${ }^{9-11}$. Clearly this might be a problem with a GA repeat-modified $I \kappa B \alpha$, as Sharipo et al. were also able to demonstrate an increase in TNF $\alpha$ induced apoptosis in transfected cells. Of greater concern, their inability to generate stable transfectants suggests that cells may be rendered sensitive to apoptosis even in the relative absence of external signals, thus limiting the usefulness of the anti-inflammatory approach. Nevertheless, the silver lining to this particular cloud might be clinical applications in promoting apoptotic responses, for example as part of therapies aimed at killing cancer cells ${ }^{10}$.

More generally, studying ways in which viruses interfere with immune pathways may eventually pay therapeutic dividends. One obvious possibility is the identification of targets for the development of new immunosuppressive drugs. However, this may not necessarily avoid the major drawback of current agents, their lack of specificity. Immunosuppressants may prevent transplant rejection or dampen down autoimmune disease, but the price to be paid is increased risk of infection or tumorigenesis. Targeting the GA repeat to specific proteins may provide one way round this, in that this strategy inhibits degradation of proteins in which the repeat is inserted but does not affect proteasome-mediated proteolysis of other proteins. As immunosuppressive strategies become smarter, this observation could be exploited. Sharipo et al. suggest one example: using the GA repeat in gene transfer therapies to suppress the problematic immune response to vector, or alternatively to inhibit the degradation of the therapeutic product. Another example might be genetically engineering the repeat into xenografts, either to suppress immunity to highly immunogenic proteins or to prevent turnover of other transgenes inserted to prolong graft survival.

There are other potential clinical applications. Why waste time designing new immunomodulatory molecules when evolution has already provided a catalogue of tried and tested proteins and motifs? These same proteins may be attractive targets for novel antiviral therapies. Viral vaccines may be made more potent by deleting genes known to inhibit the immune response to vaccine genomes. Finally, studies of host-viral interactions point to obvious sites at which to search for genetic variability influencing the host immune response.

Adapting strategies used by pathogens is an old trick, of which the best known example is antibiotics. But as molecular pathways are described in ever increasing detail, and drug design and delivery become more sophisticated, new opportunities will arise. The age-old war between host and pathogen continues, but now for the first time we can see the fine print of enemy plans. As in any conflict, the challenge is to turn this to our own advantage.

1. Sharipo, A., Imreh, M., Leochiks, A., Imreh, S. \& Masucci, M.G. A minimal Gly-Ala repeat prevents the interaction of ubiquinated IкB $\alpha$ with the proteasome: A new mechanism for selective inhibition of proteolysis. Nature Med. 4, 939-944 (1998).

2. Coux, O., Tanaka, K. \& Goldberg, A.L. Structure and functions of the $20 S$ and $26 S$ proteasomes. Annu. Rev.
Biochem. 265, 801-847 (1996)

3. Masucci, M.G. \& Ernberg, I. Adaptation to a life within the immune system. Trends Microbiol. 2, 125-130 (1994).

4. Steven, N.M. Epstein-Barr virus latent infection in vivo. Rev. Med. Virol. 7, 97-106 (1997).

5. Levitskaya, J. et al. Inhibition of antigen processing by the internal repeat region of the Epstein-Barr virus nuclear antigen-1. Nature 375, 685-688 (1995).

6. Levitskaya, J., Sharipo, A., Leonchiks, A., Ciechanover, A. \& Masucci, M.G. Inhibition of ubiquitin/proteasome-dependent protein degradation by the Gly-Ala repeat domain of the Epstein-Barr virus nuclear antigen 1. Proc. Natl. Acad. Sci. USA 94, 12616-12621 (1997).

7. Barnes, P.J. \& Karin, M. Nuclear factor $\times B$-a pivotal transcription factor in chronic inflammatory disease. New Eng. J. Med. 336, 1066-1071 (1997).

8. Bach, F.H. et al. Modification of vascular responses in xenotransplantation: Inflammation and apoptosis. Nature Med. 3, 944-948 (1997).

9. Beg, A.A. \& Baltimore, D. An essential role for NFkappa B in preventing TNF-alpha-induced cell death. Science 274, 782-784 (1996).

10. Wang, C.Y., Mayo, M.W. \& Baldwin, A.S., Jr. TNFand cancer therapy-induced apoptosis: Potentiation by inhibition of NF-kappa B. Science 274, 784-787 (1996).

11. Van Antwerp, D.J., Martin, S.J., Kafri, T., Green, D.R. \& Verma, I.M. Suppression of TNF-alpha-induced apoptosis by NF-kappa B. Science 274, 787-789 (1996).

12. Riddell, S.R. \& Greenberg, P.D. T cell therapy of human CMV and EBV infection in immunocompromised hosts. Rev. Med. Virol. 7, 181-192 (1997)

13. Karre, K. \& Welsh, R.M. Viral decoy vetoes killer cell. Nature 386, 446-447 (1997).

14. Wiertz, E.J., Mukherjee, S. \& Ploegh, H.L. Viruses use stealth technology to escape from the host immune system. Mol. Med. Today 3, 116-123 (1997).

15. Oldstone, M.B. How viruses escape from cytotoxic $T$ lymphocytes: Molecular parameters and players. Virology 234, 179-185 (1997).

16. McMichael, A. T cell responses and viral escape. Cell 93, 673-676 (1998).

Centre for Nephrology

Royal Free and University College Medical

School of University College London

Royal Free Campus

London NW3 2PF, U.K.

\title{
A2M is associated with late-onset Alzheimer disease
}

The APP, PS1 and PS2 genes are known to be associated with 30-40 percent of cases of earlyonset familial Alzheimer disease $(A D)$ but the genetic basis underlying late-onset $A D$ is less well characterized. Now, in a large cohort study, Rudy Tanzi and colleagues (Nature Genet. $19,357-360,1998$ ) have found that a polymorphic variant of the $A 2 M$ gene is more common in late-onset AD patients than in their unaffected siblings. This gene encodes $\alpha$-2macroglobulin, which binds tightly to and mediates the breakdown and clearance of amyloid- $\beta$ - the principal component of the waxy plaques characteristic of $A D$.

Whereas $A 2 M$ appears to influence the likelihood of developing late-onset $A D$, the $A P O E$ gene is widely thought to predict the age of $A D$ onset in susceptible individuals. This 'modifier' effect is now confirmed by John Breitner and co-workers (Nature Genet. 19, $321-322,1998$ ), who studied nearly 5,000 elderly individuals and found that their risk of developing $A D$ reached a plateau in late old age beyond which no further $A D$ cases were detected, regardless of APOE genotype.

Intriguingly, the products of $A 2 M, A P O E$ and $A P P$ all bind the low density lipoprotein receptor-related protein (LRP). Furthermore, the $L R P 1$ gene encoding LRP may itself be associated with late-onset AD. Together, these findings indicate that the heterogeneous collection of genetic factors involved in $A D$ appear to exert their influence through a common biological pathway.

Carina Dennis, Nature Genetics 